\title{
FOUR CLASSES OF SEPARABLE METRIC INFINITE-DIMENSIONAL MANIFOLDS ${ }^{1}$
}

\author{
BY T. A. CHAPMAN
}

Communicated by R. D. Anderson, October 6, 1969

1. Introduction. The purpose of this note is to announce some new embedding, homeomorphism, and characterization theorems regarding certain infinite-dimensional manifolds. We list these theorems below along with some of the principal known results in this area. It is expected that these new results will constitute a portion of the author's dissertation and their proofs will appear in a longer paper that is in preparation.

2. Definitions and notation. Each infinite-dimensional separable Fréchet space (and therefore each infinite-dimensional separable Banach space) is homeomorphic to $s$, the countable infinite product of open intervals $(-1,1)$ (see $[3]$ ). A Fréchet manifold (or F-manifold) is a separable metric manifold modeled on $s$. A Hilbert cube manifold (or Q-manifold) is a separable metric manifold modeled on the Hilbert cube $I^{\infty}$, which we represent as the countable infinite product of closed intervals $[-1,1]$.

Let $\sigma$ be the set consisting of all points in $s$ having at most finitely many nonzero coordinates and define a $\sigma$-manifold to be a separable metric manifold modeled on $\sigma$. Let $\Sigma$ be the set consisting of all points in $s$ having at most finitely many coordinates not in $\left[-\frac{1}{2}, \frac{1}{2}\right]$ and define a $\Sigma$-manifold to be a separable metric manifold modeled on $\Sigma$.

A subset $K$ of a space $X$ is a $Z$-set in $X$ if $K$ is closed and if for every nonnull homotopically trivial open set $U$ in $X, U \backslash K$ is nonnull and homotopically trivial.

A subset $M$ of a metric space $X$ is said to have the (finite-dimensional) compact absorption property (or $(f-d)$ cap) in $X$ provided that

(1) $M=\bigcup_{n=1}^{\infty} M_{n}$, where $M_{n}$ is a (finite-dimensional) compact $Z$-set in $X$, and

AMS Subject Classifications. Primary 5755; Secondary 5705, 5701.

Key Words and Phrases. Fréchet manifolds, Hilbert cube manifolds, Property Z, (finite-dimensional) compact absorption property, infinite deficiency.

1 This article was presented to the Society January 24, 1970.

The research was conducted while the author held a NASA traineeship. 
(2) for each (finite-dimensional) compact set $K$ in $X$, each integer $m>0$, and each $\epsilon>0$, there is an integer $n>0$ and an embedding $h: K \rightarrow M_{n}$ such that $h \mid K \cap M_{m}=\mathrm{id}$ and $d(h, \mathrm{id})<\epsilon$.

An $(f-d)$ cap-set for $X$ is a set which has the $(f-d)$ cap in $X$. We adopt the convention that the symbol $(f-d)$ cap implies two alternative conditions, one for $f-d$ cap and the other for cap. In [2] it is shown that $\sigma$ is an $f-d$ cap-set for $s$ and $\Sigma$ is a cap-set for $s$.

Let $X$ be a space and let $u$ be an open cover of $X$. A function $f: X \rightarrow X$ is said to be limited by $\mathcal{U}$ provided that for each $x \in X, x$ and $f(x)$ are both contained in some member of $\mathcal{U}$. $\mathrm{By} \mathrm{St}^{n}(\mathcal{U})$ we will mean the $n$th star of the cover $\mathcal{U}$.

As in [6], a subset $K$ of a space $X$ is said to be strongly negligible provided that given any open cover $\mathcal{U}$ of $X$, there is a homeomorphism of $X$ onto $X \backslash K$ which is limited by $น$.

A subset $K$ of $I^{\infty}$ is said to have infinite deficiency (or infinite codimension) if for each of infinitely many different coordinate directions, $K$ projects onto a single interior point of $(-1,1)$.

3. Principal results on $F, Q, \Sigma$, and $\sigma$-manifolds. The theorems are those of the author unless other authorship is denoted.

I. Characterization of MANifolds by homotopy type.

Theorem 1 (Henderson [8]). If $X$ and $Y$ are F-manifolds of the same homotopy type, then they are homeomorphic.

THEOREM 2. If $X$ and $Y$ are both $\sigma$-manifolds or both $\Sigma$-manifolds and are of the same homotopy type, then they are homeomorphic.

II. OPEN EMBEDDING THEOREMS.

Theorem 3 (Henderson [8]). If $X$ is an F-manifold, then $X$ can be embedded as an open subset of $s$.

Theorem 4. If $X$ is a $\sigma$ (or $\Sigma$ )-manifold, then $X$ can be embedded as an open subset of $\sigma$ (or $\Sigma)$.

III. Product theorems.

TheOREM 5 (West [9]). If $K$ is any countable locally-finite simplicial complex, then $|K| \times s$ is an F-manifold and $|K| \times I^{\infty}$ is a $Q-$ manifold.

THEOREM 6. If $K$ is any countable locally-finite simplicial complex, then $|K| \times \sigma$ is a $\sigma$-manifold and $|K| \times \Sigma$ is a $\Sigma$-manifold.

Theorem 7. If $X$ is a $\sigma$-manifold and $Y$ is a $\Sigma$-manifold of the same homotopy type, then $X \times I^{\infty}$ and $Y$ are homeomorphic. 
IV. FACTOR THEOREMS.

Theorem 8 (Henderson [8]). If $X$ is an F-manifold, then there is a countable locally-finite simplicial complex $K$ such that $X$ and $|K| \times s$ are homeomorphic.

THEOREM 9. If $X$ is a $\sigma$ (or $\Sigma$ )-manifold, then there is a countable locally-finite simplicial complex $K$ such that $X$ is homeomorphic to $|K| \times \sigma($ or $|K| \times \Sigma)$.

Theorem 10 (Anderson ANd Schori [5]). If $X$ is an F-manifold, then $X, X \times s$, and $X \times I^{\infty}$ are all homeomorphic.

Theorem 11 (Anderson ANd Schori [5]). If $X$ is a Q-manifold, then $X$ and $X \times I^{\infty}$ are homeomorphic.

Theorem 12. If $X$ is a $\sigma$-manifold and $I^{n}$ is any $n$-cell, then $X$, $X \times \sigma$, and $X \times I^{n}$ are all homeomorphic.

Theorem 13. If $X$ is any $\Sigma$-manifold, then $X, X \times \Sigma$, and $X \times I^{\infty}$ are all homeomorphic.

V. Relationships BETWEEN $F, Q, \Sigma$, AND $\sigma$-MANIFOLdS.

Theorem 14. If $X$ is a $\sigma$ (or $\Sigma$-manifold), then $X$ can be embedded as an $f$-d cap (or cap)-set for an $F$-manifold and also for a $Q$-manifold.

Theorem 15 (ANDERson [2]). If $X$ is $I^{\infty}$ or $s$ and $M, N$ are $(f-d)$ cap-sets for $X$, then there is a homeomorphism of $X$ onto itself taking $M$ onto $N$.

THEOREM 16. If $X$ is an $F$ or $Q$-manifold and $M$ is an $f-d$ cap (or cap)-set for $X$, then $M$ is a $\sigma$ (or $\Sigma$ )-manifold.

THeOREM 17. If $X$ is an $F$ or $Q$-manifold, $M$ and $N$ are $(f-d)$ cap-sets for $X$, and $\mathcal{U}$ is an open cover of $X$, then there is a homeomorphism of $X$ onto itself which takes $M$ onto $N$ and is limited by $\mathcal{u}$.

TheOREM 18 (ANDERson [2]). If $M$ is an $(f-d)$ cap-set for $I^{\infty}$, then $I^{\infty} \backslash M$ is homeomorphic to $s$.

THEOREM 19. If $X$ is any $Q$-manifold and $M$ is an $(f-d)$ cap-set for $X$, then $X \backslash M$ is an F-manifold which is of the same homotopy type as $X$.

VI. Subsets AND SUPERSETS OF $F, Q, \Sigma$, AND $\sigma$-MANifolds.

Theorem 20 (Anderson, Henderson and West [6]). A necessary and sufficient condition that a closed subset $K$ of an $F$-manifold be a $Z$-set is that $K$ be strongly negligible. 
THEOREM 21. A necessary and sufficient condition that a closed subset $K$ of $a \sigma$ or $\Sigma$-manifold be $a Z$-set is that $K$ be strongly negligible.

THEOREM 22. Let $M$ be an $(f-d)$ cap-set for an $F$ or $Q$-manifold $X$ and let $K$ be a $Z$-set in $X$. Then $M \backslash K$ is an $(f-d)$ cap-set for $X$.

THEOREM 23. Let $M$ be an $(f-d)$ cap-set for an $F$ or $Q$-manifold $X$ and let $K$ be a countable union of (finite-dimensional) compact Z-sets in $X$. Then $M \cup K$ is an $(f-d)$ cap-set for $X$.

VII. HomeOMORPHISM EXTENSION THEOREMS.

Theorem 24 (Anderson AND McCharen [4]). Let $X$ be an $F$-manifold, let $K_{1}, K_{2}$ be $Z$-sets in $X$, let $u$ be an open cover of $X$, and let $h$ be a homeomorphism of $K_{1}$ onto $K_{2}$ such that there is a homotopy $H: K_{1} \times I \rightarrow X$ for which $H_{0}=\mathrm{id}, H_{1}=h$, and $H(\{x\} \times I)$ is contained in some member of $u$, for each $x \in K_{1}$. Then $h$ can be extended to a manifold homeomorphism which is limited by $\mathrm{St}^{4}(\mathcal{u})$.

Theorem 25. Let $X$ be a $\sigma$ or $\Sigma$-manifold, let $K_{1}, K_{2}$ be $Z$-sets in $X$, let $u$ be an open cover of $X$, and let $h$ be a homeomorphism of $K_{1}$ onto $K_{2}$ such that there is a homotopy $H: K_{1} \times I \rightarrow X$ for which $H_{0}=\mathrm{id}, H_{1}=h$, and $H(\{x\} \times I)$ is contained in some member of $u$, for each $x \in K_{1}$. Then $h$ can be extended to a manifold homeomorphism which is limited by $\mathrm{St}^{28}(\mathfrak{u})$.

VIII. Complete EXtensions of $\Sigma$ AND $\sigma$-MANifolds.

TheOREM 26. Let $X$ be a $\sigma$ (or $\Sigma$ )-manifold and let $Y$ be a complete separable metric space containing $X$. Then there is an F-manifold $Z$ such that $X \subset Z \subset Y$ and $X$ is an $f-d$ cap (or cap)-set for $Z$.

IX. INFINITE DEFICIENCY.

TheOREM 27 (ANDERson [1]). Let $X$ be $I^{\infty}$ or $s$ and let $K$ be a closed subset of $X . A$ necessary and sufficient condition that $K$ be a $Z$-set in $X$ is that there exists a homeomorphism of $X$ onto itself taking $K$ onto a set having infinite deficiency.

TheOREM 28 (ChAPMAN [7]). Let $X$ be an F-manifold and let $K$ be $a$ closed subset of $X$. A necessary and sufficient condition that $K$ be a $Z$-set in $X$ is that there exists a homeomorphism $h$ of $X$ onto $X \times s$ such that $\pi . \circ h(K)$ has infinite deficiency.

Theorem 29. Let $X$ be a $Q$-manifold and let $K$ be a closed subset of $X$. A necessary and sufficient condition that $K$ be a $Z$-set in $X$ is that there exists a homeomorphism $h$ of $X$ onto $X \times I^{\infty}$ such that $\pi_{I^{\infty}} \circ h(K)$ has infinite deficiency. 


\section{REFERENCES}

1. R. D. Anderson, On topological infinite deficiency, Michigan Math. J. 14 (1967), 365-383. MR 35 \#4893.

2. - On dense sigma-compact subsets of infinite-dimensional spaces, Trans. Amer. Math. Soc. (to appear).

3. R. D. Anderson and R. H. Bing, A complete elementary proof that Hilbert space is homeomorphic to the countable infinite product of lines, Bull. Amer. Math. Soc. 74 (1968), 771-792. MR 37 \#5847.

4. R. D. Anderson and John D. McCharen, On extending homeomorphisms to Fréchet manifolds, Proc. Amer. Math. Soc. (to appear).

5. R. D. Anderson and R. Schori, Factors of infinite-dimensional manifolds, Trans. Amer. Math. Soc. 142 (1969), 315-330.

6. R. D. Anderson, David W. Henderson and James E. West, Negligible subsets of infinite-dimensional manifolds, Compositio Math. 21 (1969), 143-150.

7. T. A. Chapman, Infinite deficiency in Fréchet manifolds, Trans. Amer. Math. Soc. (to appear).

8. David W. Henderson, Infinite-dimensional manifolds are open subsets of Hilbert space, Bull. Amer. Math. Soc. 75 (1969), 759-762.

9. James E. West, Infinite products which are Hilbert cubes, Trans. Amer. Math. Soc.

Louisiana State University, Baton Rouge, Louisiana 70803 